\title{
Clinical perspective on aztreonam lysine for inhalation in patients with cystic fibrosis
}

This article was published in the following Dove Press journal:

Infection and Drug Resistance

27 October 2010

Number of times this article has been viewed

\author{
Martha K Daddario' \\ Jennifer K Hagerman ${ }^{2}$ \\ Michael E Klepser ${ }^{2}$ \\ 'Spectrum Health, Grand Rapids, \\ Michigan; ${ }^{2}$ Ferris State University \\ College of Pharmacy, Kalamazoo, \\ Michigan, USA
}

Correspondence: Michael E Klepser Ferris State University College of Pharmacy, I000 Oliver Lane, Kalamazoo, MI 49008, USA

Email michaelklepser@ferris.edu

\begin{abstract}
Progressive obstructive lung disease is a characteristic component of cystic fibrosis (CF). It is the pulmonary manifestations, including obstruction and endobronchial infection, which directly contribute to the premature mortality of patients affected with CF. Due to the devastating effects on the pulmonary system, interest abounds in ways to improve antimicrobial delivery to the lungs and to impact clinical patient outcomes positively, whilst minimizing systemic toxicities. Recently, aztreonam lysine for inhalation solution, a new monobactam formulation, was approved by the US Food and Drug Administration for use in a subgroup of CF patients with Pseudomonas aeruginosa to improve respiratory symptoms. The purpose of this review is to present a summary of relevant pharmacologic, microbiologic, and clinical data related to the use of aztreonam lysine for inhalation in patients with CF.
\end{abstract}

Keywords: aztreonam, cystic fibrosis, inhaled, aerosolized, antibiotic, Pseudomonas aeruginosa

\section{Introduction}

Cystic fibrosis $(\mathrm{CF})$ is an autosomal recessive genetic disease characterized by multiple organ system dysfunction, primarily affecting the pulmonary and gastrointestinal systems. The Cystic Fibrosis Foundation reports that approximately 30,000 children and adults in the US and 70,000 patients worldwide are afflicted with $\mathrm{CF}{ }^{1}$ This roughly translates into one in every 3500 children in the US being born with $\mathrm{CF}^{1}$ The incidence of CF is greatest among Caucasians and substantially less in other races, affecting one in 17,000 blacks and one in 90,000 Asians. ${ }^{1}$

A hallmark symptom of CF is progressive obstructive lung disease. ${ }^{2-5}$ Pulmonary obstruction contributes to the development of chronic endobronchial infections among patients with CF. Complications arising from these infectious processes are associated with nearly $85 \%$ of CF-related mortality. ${ }^{2}$ Mutations in the CF transmembrane conductance regulator protein lead to impairment of $\mathrm{Cl}^{-}, \mathrm{Na}^{+}$, and water transport in the airways, intestinal epithelia, sweat ducts, and proximal intestine. In the lungs, this leads to decreased fluid volume being secreted into the mucus. This results in the production of "dehydrated", thick, and sticky mucus. In turn, this abnormal mucus is relatively resistant to ciliary clearance and elimination by cough. The stagnated mucus in the airways is prone to bacterial colonization which can lead to infection. ${ }^{3-5}$ Recurrent endobronchial infections and the exaggerated inflammatory response ultimately result in progressive endobronchial obstruction and destruction of lung parenchyma, leading to loss of lung function..$^{2-5}$ Although various bacteria can be cultured from the 
mucus of patients with $\mathrm{CF}$, approximately $80 \%$ of patients become chronically infected with Pseudomonas aeruginosa by adulthood. ${ }^{1}$ Chronic colonization and recurrent infection with $P$. aeruginosa, particularly the mucoid phenotype, are associated with weight loss, accelerated decline in pulmonary function, and premature mortality. ${ }^{6-9}$

Recognition of the link between colonization and repeated infections with $P$. aeruginosa and deterioration of pulmonary function has ushered in an era of improved patient care. The standard of care for patients with $\mathrm{CF}$, as it pertains to bacterial colonization/infection, focuses on two issues, ie, maintaining clear airways and controlling bacterial density. Maintenance of clear airways is typically achieved through the use of several modalities, including physiotherapy, inhaled hypertonic saline, and inhaled dornase alfa. ${ }^{1,2,10}$ Additionally, azithromycin may be administered as an adjuvant therapy to decrease airway inflammation. ${ }^{2}$ The second aspect of care, decreasing bacterial colonization and fighting infection, is accomplished via the administration of systemic and inhaled antimicrobials. ${ }^{2,11}$ As a result of these efforts, the proportion of patients with preserved lung function and the predicted median age of survival have increased since $1985 .{ }^{1}$ Unfortunately, despite these gains, CF-related morbidity and mortality remain unacceptably high.

Appropriate antibiotic therapy targeted against colonizing/infecting bacteria, especially $P$. aeruginosa, is an essential strategic component of preservation of lung function among patients with CF. Regimens consisting of intravenous, oral, and/or inhaled formulations of several classes of antibiotics including aminoglycosides, $\beta$-lactams, polymyxins, and fluoroquinolones may have the ability to reduce bacterial densities in sputum. However, evidence linking these reductions to sustained improvement or preservation of lung function is generally lacking. Owing to the need for longterm administration to maintain bacterial suppression, drug toxicity, patient compliance, and emergence of resistance can be significant therapeutic challenges. ${ }^{12}$ Aerosolized administration of antibiotics has been advocated for the management of endobronchial colonization/infection in patients with $\mathrm{CF}^{5}$ Inhalation of antimicrobials is appealing because it can deliver high concentrations of antibiotics directly to the airways while minimizing systemic bioavailability and toxicity. Furthermore, delivery of high antibiotic concentrations to the lungs may overwhelm the inhibitory effects of purulent sputum on antibacterial activity and suppress the emergence of resistance..$^{5,13,14}$ Delivery of antibiotics by inhalation may also improve patient adherence to therapies by minimizing the need for parenteral administration.
In the setting of $\mathrm{CF}$, aerosolized antibiotics have been utilized in three general settings, ie, chronic suppressive therapy in patients with stable lung function, treatment for colonized patients with suboptimal lung function, and treatment of acute symptomatic pulmonary exacerbations. Until recently, tobramycin inhalation solution $\left(\mathrm{TOBI}^{\circledR}\right)$ was the only aerosolized antibiotic approved by the US Food and Drug Administration (FDA). Currently, tobramycin inhalation solution is the sole aerosolized antibiotic recognized by the Cystic Fibrosis Foundation to improve lung function and lung health in patients with $\mathrm{CF}^{2}$ Tobramycin inhalation solution may be considered as maintenance therapy to reduce exacerbations of $P$. aeruginosa infections in $\mathrm{CF}$ patients six years of age and older with persistent $P$. aeruginosa positive cultures and asymptomatic, mild, or moderate to severe lung disease. ${ }^{2,15}$ Despite this endorsement, lingering concerns regarding long-term toxicity, development of resistance, and diminished bactericidal activity in the presence of purulent sputum persist. ${ }^{13,14,16-18}$

Interest in the development of aerosolized antibiotics for use among patients with CF remains high. Several additional aerosolized antimicrobial formulations are under investigation including liposomal, liquid, or dry powder formulation of antibiotics, such as a tobramycin-fosfomycin combination, amikacin, gentamicin, ciprofloxacin, levofloxacin, colistin, azithromycin, and vancomycin. ${ }^{19-23}$ Aztreonam for inhalation (Cayston ${ }^{\circledR}$; Gilead, Foster City, CA, USA) was recently approved by the FDA for improvement of respiratory symptoms in CF patients with $P$. aeruginosa who are aged seven years and older with forced expiratory volume in one second $\left(\mathrm{FEV}_{1}\right)$ 25\%-75\% predicted. ${ }^{24}$ This review was undertaken to summarize and present relevant pharmacologic, microbiologic, and clinical data regarding this formulation. Additionally, we have attempted to provide insight into pharmacoeconomic and quality of life considerations. Data for this review were gathered following a comprehensive literature search of English language publications in the PubMed database.

\section{Pharmacology}

Aztreonam belongs to the monobactam class of antibiotics. It is a bactericidal agent that exhibits time-dependent pharmacodynamics, similar to those of the beta-lactams. The mechanism of action of aztreonam is exerted through the inhibition of bacterial cell wall synthesis secondary to binding to penicillin-binding proteins. ${ }^{24}$ Aztreonam for injection (Azactam ${ }^{\circledR}$; Bristol-Myers Squibb, Princeton, NY, USA) is currently available as an intravenous formulation, and is 
approved for the treatment of complicated and uncomplicated urinary tract infections, intra-abdominal infections, skin and soft tissue infections, lower respiratory infections, septicemia, and gynecologic infections caused by susceptible Gram negative organisms. ${ }^{25}$

The formulation for injection is prepared as an arginine salt. It has been reported that this salt may be a substrate for the production of nitric oxide in the lung, which may potentiate airway inflammation and induce bronchoconstriction. ${ }^{26,27}$ In an attempt to circumvent this problem, a lysine salt of aztreonam was developed specifically for inhalational use. It should be noted, however, that clinical complications as a result of aerosolized administration of aztreonam arginine have not been reported.

Aztreonam for inhalation is available as a single-use vial containing $75 \mathrm{mg}$ of aztreonam and $46.7 \mathrm{mg}$ of lysine. The lyophilized powder is to be reconstituted with $1 \mathrm{~mL}$ of sterile preservative-free diluent, and administered using the Altera ${ }^{\circledR}$ Nebulizer System (PARI Pharma, Midlothian, VA, USA). ${ }^{24}$ The inhalational formulation of aztreonam has a $\mathrm{pH}$ range 4.5-6.0 once reconstituted. The antimicrobial activity of aztreonam is not affected over a $\mathrm{pH}$ range $6-8$, the presence of human serum, or reduced oxygen tensions. ${ }^{24,25}$ Aztreonam for inhalation should be stored refrigerated at $2^{\circ} \mathrm{C}$ to $8^{\circ} \mathrm{C}$ until needed; however, it is stable at room temperature for up to 28 days. Once reconstituted, aztreonam for inhalation should be used immediately. ${ }^{24}$

\section{Microbiology}

Aztreonam has potent in vitro activity against a wide spectrum of aerobic Gram negative organisms, including P. aeruginosa, Escherichia coli, Haemophilus influenzae, Proteus mirabilis, Citrobacter spp, Enterobacter spp, Klebsiella spp, and Serratia spp. ${ }^{24,25}$ Additionally, susceptibility to aztreonam has been documented for strains of $P$. aeruginosa recovered from patients with $\mathrm{CF}^{28}$ In this study, 1240 isolates of $P$. aeruginosa were recovered from the sputum of 508 patients with $\mathrm{CF}$ and in vitro susceptibilities were determined for aztreonam and six other agents. ${ }^{28}$ Against this bank of isolates, the $\mathrm{MIC}_{50}$ and $\mathrm{MIC}_{90}$ for aztreonam were $\leq 2 \mu \mathrm{g} / \mathrm{mL}$ and $32 \mu \mathrm{g} / \mathrm{mL}$, respectively. Additionally, using interpretive breakpoints, only $11.9 \%$ of isolates were classified as being resistant to aztreonam. Against these same isolates, only $5.4 \%$ of isolates were categorized as resistant to tobramycin. It must be noted that application breakpoints for the treatment of pulmonary infections may be misleading, especially if novel routes of administration such as aerosolization are employed. Furthermore, comparison between agents may be skewed because isolates were not tested in the presence of sputum from $\mathrm{CF}$ patients. Aztreonam is much less active against Burkholderia cepacia and Stenotrophomonas maltophilia. ${ }^{29-31}$ Aztreonam does not possess activity against aerobic Gram positive organisms or anaerobic organisms.

In order to determine possible detrimental effects of nebulization on the activity of aztreonam lysinate, susceptibility testing was performed with pre- and postnebulization aztreonam lysinate and compared with MICs determined for aztreonam arginate. MICs were determined for a variety of multidrug-resistant clinical isolates, including $P$. aeruginosa, B. cepacia complex, S. maltophilia, Achromobacter xylosoxidans, and Staphylococcus aureus. ${ }^{31}$ Based on attainable levels of aztreonam in sputum following nebulization and MIC distributions, the authors concluded that both salt formulations of aztreonam would exhibit good activity against $B$. cepacia complex and multidrug-resistant $P$. aeruginosa. The $\mathrm{MIC}_{50}$ values for aztreonam lysinate against P. aeruginosa were $8 \mu \mathrm{g} / \mathrm{mL}$ and $4 \mu \mathrm{g} / \mathrm{mL}$, pre- and postnebulization, respectively. The $\mathrm{MIC}_{90}$ values against $P$. aeruginosa were $512 \mu \mathrm{g} / \mathrm{mL}$ and $1024 \mu \mathrm{g} / \mathrm{mL}$, pre- and postnebulization, respectively. Against $B$. cepacia complex isolates, the pre- and postnebulization $\mathrm{MIC}_{50}$ was $32 \mu \mathrm{g} / \mathrm{mL}$. Aztreonam exhibited little activity against $S$. maltophilia $\left(\mathrm{MIC}_{50} \geq 1024 \mu \mathrm{g} / \mathrm{mL}\right)$ and $A$. xylosoxidans $\left(\mathrm{MIC}_{50}=1024 \mu \mathrm{g} / \mathrm{mL}\right)$. Comparison of pre- and postnebulization MICs revealed that $82 \%$ of isolates exhibited MICs that were either identical or within one dilution of each other pre- and postnebulization. Therefore, it was concluded that nebulization did not appreciably affect the in vitro activity of aztreonam lysinate. Furthermore, there were no differences with respect to the in vitro activities of the aztreonam lysinate and arginate salts.

The effect of sputum from patients with CF on the killing activity of aztreonam has also been studied. Time-kill studies were conducted using $1 \%$ pooled sterile sputum collected from patients with $\mathrm{CF}$ or $10 \%$ porcine gastric mucin and compared with controls (growth medium only). ${ }^{29}$ Kill curves were generated following exposure of a strain of $P$. aeruginosa to aztreonam or tobramycin at concentrations equal to $0,0.1,1.0$, or 10 times the MIC. Upon inspection of time-kill data it was determined that the bactericidal activity of aztreonam was not affected by either sputum or mucin. In contrast, testing conducted with tobramycin in the presence of sputum and mucin resulted in reduced activity, generally $2-3 \log _{10}$ less killing.

\section{Pharmacokinetics}

Gibson et al conducted a multicenter, double-blind, placebo-controlled, dose-escalation trial to determine the 
pharmacokinetics and tolerability of nebulized aztreonam lysinate. ${ }^{31}$ Thirty-five patients with previously diagnosed CF, including 18 adults $\geq 18$ years of age and 17 adolescents $>12$ and $<18$ years of age were randomized to receive a single dose of $75 \mathrm{mg}, 150 \mathrm{mg}$, and $225 \mathrm{mg}$ aztreonam lysinate on study days 0,1 , and 2 , respectively, or placebo via an eFlow $^{\circledR}$ Electronic Nebulizer (PARI Pharma). All patients were required to have an $\mathrm{FEV}_{1} \geq 40 \%$ predicted, oxygen saturation $\geq 90 \%$ on room air, and be clinically stable, with no acute upper or lower respiratory tract infection. Plasma and sputum samples were collected for analysis following nebulized administration of aztreonam or placebo.

Sputum samples were collected from patients at $10 \mathrm{~min}-$ utes, two hours, and four hours postnebulization of the assigned study dose of aztreonam on study days 0,1 , and $2 .{ }^{31}$ The median sputum concentrations were highest 10 minutes postnebulization in both the adult and adolescent groups. For adults, the median sputum concentrations at four hours postnebulization were $16 \mu \mathrm{g} / \mathrm{g}, 36 \mu \mathrm{g} / \mathrm{g}$, and $52 \mu \mathrm{g} / \mathrm{g}$, in the $75 \mathrm{mg}, 150 \mathrm{mg}$, and $225 \mathrm{mg}$ aztreonam groups, respectively (Figure 1). In the adolescent cohort, the median sputum concentrations four hours postnebulization were $2 \mu \mathrm{g} / \mathrm{g}, 7 \mu \mathrm{g} / \mathrm{g}$, and $8 \mu \mathrm{g} / \mathrm{g}$, in the $75 \mathrm{mg}, 150 \mathrm{mg}$, and $225 \mathrm{mg}$ aztreonam groups, respectively (Figure 2). Aztreonam concentrations in adolescent patients were found to be consistently lower than those in adults. In fact, in contrast with adults, sputum concentrations of aztreonam in the adolescent cohort did not exhibit a dose-related increase at any time point. The authors speculated this finding to be attributable to increased salivary dilution in airway secretions due to milder lung disease, reduced central airway deposition of aztreonam, and increased lung clearance of aztreonam compared with adults. Based on these data, the authors concluded that aztreonam concentrations measured in sputum immediately following single doses of aztreonam lysinate generally exceed the aztreonam $\mathrm{MIC}_{90}(32 \mu \mathrm{g} / \mathrm{mL})$ for $P$. aeruginosa by at least 10-fold. ${ }^{31}$

Plasma pharmacokinetics of aztreonam were characterized following nebulized administration of a $75 \mathrm{mg}$ dose in the adult cohort $(\mathrm{n}=12) \cdot{ }^{31}$ A mean maximum plasma concentration for aztreonam of $419 \mathrm{ng} / \mathrm{mL}$ was noted approximately one hour following administration of the nebulized dose. The mean plasma half-life was reported to be 2.1 hours and the mean clearance was $853 \mathrm{~mL} /$ minute. Although aztreonam was detectable for at least eight hours after nebulization, overall absorption and plasma exposure was deemed to be low. ${ }^{31}$

The elimination of aerosolized aztreonam from the lungs has not been well described. Unabsorbed drug may be eliminated through sputum expectoration. The portion of drug that reaches the systemic circulation undergoes minimal metabolism and is eliminated renally through active tubular

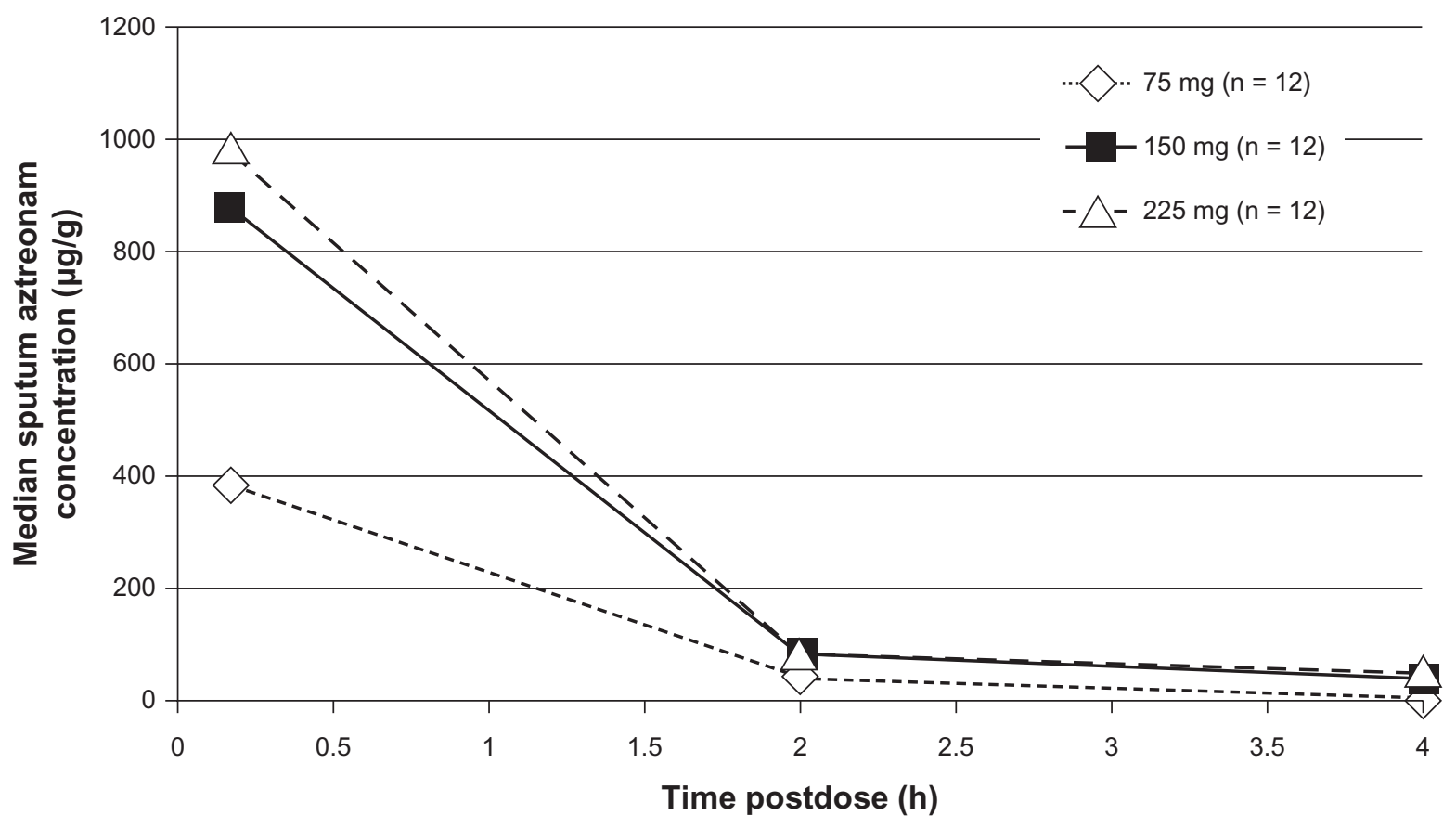

Figure I Median sputum aztreonam lysinate concentrations following nebulized administration of aztreonam to adult patients with cystic fibrosis. ${ }^{31}$ 


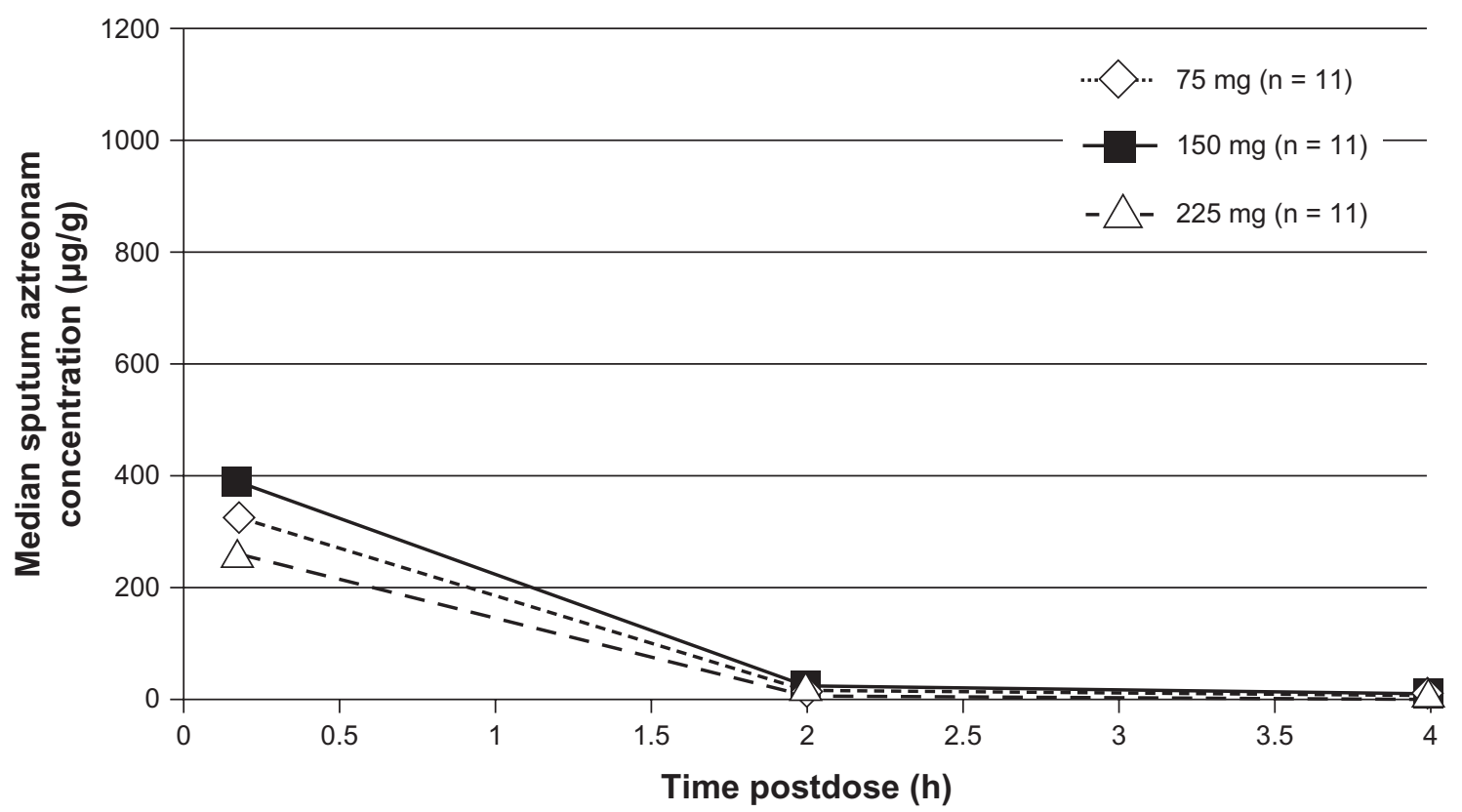

Figure 2 Median sputum aztreonam lysate concentrations following nebulized administration of aztreonam to adolescent patients with cystic fibrosis. ${ }^{31}$

secretion and glomerular filtration, with approximately $10 \%$ of the dose excreted unchanged in the urine..$^{25}$ The elimination half-life of aztreonam lysinate is approximately 2.1 hours, similar to that of parenteral aztreonam arginate. ${ }^{31}$

\section{Efficacy}

The efficacy of aztreonam lysine for inhalation (AZLI) in CF patients with stable lung function colonized/infected with $P$. aeruginosa was examined in a Phase II, randomized, double-blind, placebo-controlled trial. ${ }^{32}$ One hundred and five patients aged $\geq 13$ years with diagnosed $\mathrm{CF}, \mathrm{FEV}_{1}$ $\geq 40 \%$ predicted, oxygen saturation $\geq 90 \%$ on room air, and positive $P$. aeruginosa sputum cultures were randomized to receive $75 \mathrm{mg}$ AZLI, $225 \mathrm{mg}$ AZLI, or placebo via the eFlow ${ }^{\circledR}$ electronic nebulizer twice daily for 14 days. Randomization was stratified by severity of disease $\left(\mathrm{FEV}_{1} 40 \%\right.$ to $<60 \%$ or $\mathrm{FEV}_{1} \geq 60 \%$ ). The planned primary endpoint was the percent change in $\mathrm{FEV}_{1}$ at day 14. Mean $\mathrm{FEV}_{1}$ reported at baseline was $76.2 \%, 74.3 \%$, and $81.2 \%$ for the placebo, $75 \mathrm{mg}$, and $225 \mathrm{mg}$ groups, respectively, indicating relatively preserved lung function. At day 14, improvement in $\mathrm{FEV}_{1}$ for patients treated with AZLI $75 \mathrm{mg}$ and $225 \mathrm{mg}$ did not differ from placebo. A post hoc analysis revealed that in a subgroup of patients with a baseline $\mathrm{FEV}_{1}<75 \%$ predicted, statistically significant improvement was seen in $\mathrm{FEV}_{1}$ with $225 \mathrm{mg}$ AZLI compared with placebo at day $7(P=0.014)$. However, the improvement in $\mathrm{FEV}_{1}$ was not maintained at day 14 , and the difference from placebo was no longer statistically significant. At follow-up 14 days from the end of therapy (day 28), the mean $\mathrm{FEV}_{1}$ approached baseline in both AZLI treatment groups. ${ }^{32}$ The change from baseline in P. aeruginosa sputum density measured at days 7,14 , and 28 was also examined. P. aeruginosa sputum density increased from baseline in the placebo group at all three time points, whereas bacterial density decreased a statistically significant extent in both treatment groups at days 7 and 14 (Table 1). However, complete eradication was rare. At the end of the study period (day 28) the density of $P$. aeruginos $a$ in the sputum of AZLI-treated patients had trended back to baseline. Emergence of resistant isolates of $P$. aeruginosa over the course of the study was not observed. ${ }^{32}$ Factors which may have attributed to the failure to note a statistically significant improvement in lung function despite the noted change in bacterial density include the fact that most of the enrolled patients had relatively good lung function at baseline and the short duration of treatment and observation periods. One intriguing point noted was that patients treated with the $225 \mathrm{mg}$ dose of AZLI who were receiving bronchodilators had greater improvements in $\mathrm{FEV}_{1}$ and reductions in sputum P. aeruginosa density at day 14 compared with those who were not receiving bronchodilators. However, these differences were not maintained at day 28. It was proposed that bronchodilator-induced airway dilation may have facilitated aztreonam deposition in the lungs. ${ }^{32}$ As such, it is recommended that a bronchodilator be utilized prior to nebulized administration of aztreonam lysine. ${ }^{24}$ 
Table I Microbiologic endpoints following administration of aztreonam lysine for inhalation to patients with cystic fibrosis for 14 days $^{32}$

\begin{tabular}{|c|c|c|c|}
\hline & \multicolumn{3}{|c|}{$\begin{array}{l}\text { Mean change (SD) in } \log _{10} \text { PA cfu in sputum } \\
\text { from baseline }\end{array}$} \\
\hline & Day 7 & Day 14 & Day 28 \\
\hline Placebo & $+0.24(1.31)$ & $+0.11(0.92)$ & $+0.20(1.07)$ \\
\hline $\begin{array}{l}\text { AZLI } 75 \mathrm{mg} \\
\text { twice daily }\end{array}$ & $-1.44^{*}(1.5 \mathrm{I})$ & $-1.46 *(1.74)$ & $-0.39(1.47)$ \\
\hline $\begin{array}{l}\text { AZLI } 225 \mathrm{mg} \\
\text { twice daily }\end{array}$ & $-1.95 *(1.42)$ & $-2.22 *(1.92)$ & $-0.37(1.7)$ \\
\hline
\end{tabular}

The efficacy of AZLI for suppression of $P$. aeruginosa colonization/infection was examined among a population of patients with CF who frequently used antibiotics for P. aeruginosa airway colonization/infection. ${ }^{33}$ Patients with a diagnosis of $\mathrm{CF}$ were enrolled if they were six years of age or older and had a current $P$. aeruginosa colonization/infection diagnosed by identification of bacteria in expectorated sputum or throat culture, had three or more treatment courses with tobramycin inhalation solution in the previous year, had an $\mathrm{FEV}_{1} 25 \%-75 \%$ predicted, and an oxygen saturation of $>90 \%$ on room air. On enrollment in AIR CF-2, patients were treated with 28 days of tobramycin inhalation solution and then randomized to receive AZLI $75 \mathrm{mg}$ twice daily, AZLI $75 \mathrm{mg}$ three times daily, or placebo. The primary study endpoint was the time required before the need for additional inhaled or intravenous antipseudomonal antibiotics. Secondary endpoints included change in clinical symptoms (Cystic Fibrosis Questionnaire-Revised [CFQ-R] respiratory score), change in pulmonary function, P. aeruginosa density in sputum, and time to hospitalization. A total of 211 patients ( $78 \%$ of whom were 18 years of age or older) completed treatment with tobramycin inhalation solution and entered the randomized phase of the study. Over the 84-day follow-up, 121 patients discontinued participation from the study, 76 of which met the primary endpoint. A total of 81 patients required additional antipseudomonal antibiotics, 38 in the placebo group, 19 in the AZLI twice daily group, and 24 in the AZLI three times daily group. The median time for additional antipseudomonal antibiotic administration was $>92$ days in the AZLI twice daily group ( $P=0.002$ compared with placebo [71 days]) and 87 days in the AZLI three times daily group $(P=0.182)$. The median pooled (AZLI twice daily group and AZLI three times daily group) time to inhaled or intravenous additional antipseudomonal antibiotic administration was 21 days longer for the AZLI group than for the placebo group (92 versus 71 days, $P=0.007$ ). With respect to secondary endpoints, the adjusted mean CFQ-R respiratory scores increased by 5.01 points in the AZLI-pooled group compared with placebo (95\% confidence interval [CI]: 0.81-9.21; $P=0.020$ ). Also, an adjusted mean improvement of 6.3\% (95\% CI: 2.5-10.1; $P=0.001)$ in $\mathrm{FEV}_{1}$ among the AZLI-pooled group compared with placebo at day 28 was noted. Furthermore, the adjusted mean sputum $P$. aeruginosa density in the AZLI pooled group compared with placebo at day 28 was $-0.66 \log _{10}$ P. aeruginosa $\mathrm{cfu} / \mathrm{g}$ (95\% CI: -1.13 to $-0.19, P=0.006$ ). However, time to first hospitalization and median days per number of patients hospitalized were not statistically different between study groups. ${ }^{33}$

The finding that lung function improved following administration of AZLI despite administration to patients that averaged 5.3 courses of tobramycin inhalation solution per year was surprising. This suggests that there may be an opportunity to impact pulmonary function by administering different antimicrobials. Future trials to study the effect of antibiotic cycling may be warranted. It must be noted that data in this trial were examined following only one cycle of AZLI, and that most analyses were performed at the end of treatment. ${ }^{33}$ Furthermore, patients in the present study had relatively preserved lung function at enrollment (mean $\mathrm{FEV}_{1}$ $55 \%$ predicted) and were slightly older (mean age 26 years) than those reported in previous studies of tobramycin inhalation solution in patients with $\mathrm{CF}^{33-35}$ These differences may limit the ability to make direct comparisons with respect to study endpoints.

Another study (AIR CF-1) evaluated the use of AZLI in patients with $\mathrm{CF}$ who were six years of age or older, had moderate-to-severe lung disease $\left(\mathrm{FEV}_{1} 25 \%-75 \%\right.$ predicted), and were colonized/infected with P. aeruginosa. ${ }^{36}$ Patients were randomized to receive either AZLI $75 \mathrm{mg}$ three times daily or placebo for 28 days. The primary study endpoint for the study was change in CFQ-R respiratory score. Secondary endpoints included changes in pulmonary function, hospitalizations, and sputum $P$. aeruginosa density. One hundred and sixty-four patients were enrolled in the study. The majority of study participants (77.4\%) were 18 years of age or older, and the mean age was 29.6 years. At the end of treatment on day 28 the CFQ-R respiratory score had increased for AZLI-treated patients and decreased in those in the placebo group. This difference was noted to be clinically and statistically significant (CFQ-R respiratory 
score difference of 9.7 points, $P<0.001$, Table 2). ${ }^{36,37}$ Although the difference in CFQ-R respiratory score diminished somewhat at the follow-up on day 42 , scores were still above baseline for AZLI-treated patients, whilst scores among patients treated with placebo continued to decline (Table 2). Comparisons between patients with moderate or severe lung disease revealed the effects of treatment with AZLI to be similar. Additionally, treatment effects were greater in patients under 18 years of age. The adjusted mean $\mathrm{FEV}_{1}$ increased among AZLI-treated patients and decreased among those in the placebo group (day 28 treatment difference, $10.3 \%$; 95\% CI: 6.3-14.3; $P<0.001$ ). Improvement in $\mathrm{FEV}_{1}$ persisted until the 14-day follow-up two weeks after treatment. Conversely, $\mathrm{FEV}_{1}$ continued to drop in the placebo group (day 42 treatment difference, $5.7 \%$; 95\% CI: $2.1-9.4 ; P=0.002)$. The authors did note that there was a modest correlation between CFQ-R respiratory scores and $\mathrm{FEV}_{1}$ values, thus suggesting that patient reporting of symptoms do not always correlate with pulmonary function. Adjusted mean sputum P. aeruginosa density declined in the AZLI treatment group with minimal change in the placebo group (day 28 treatment difference, $-1.453 \log _{10} \mathrm{cfu} / \mathrm{g} ; 95 \%$ CI: -2.1 to $-0.8 ; P<0.001)$. Within two weeks following the end of treatment, density values were near baseline for both groups. Although there was a trend toward reduction in need for hospitalization among the AZLI-treated patients ( $5 \%$ versus $14 \%$ in the placebo group), this difference did not reach statistical significance $(P=0.064) .{ }^{36}$ In this study, the majority of patients were adults (mean age 29.6 years) and had moderate lung disease (mean $\mathrm{FEV}_{1} 54 \%$ of

Table 2 Endpoint results from the Retsch-Bogart et al trial ${ }^{36,37}$

\begin{tabular}{|c|c|c|c|}
\hline Endpoint & $\begin{array}{l}\text { AZLI tid } \\
(n=80)\end{array}$ & $\begin{array}{l}\text { Placebo } \\
(n=84)\end{array}$ & $P$ value \\
\hline \multicolumn{4}{|c|}{ CFQ-R respiratory scores, change from baseline (mean $\pm S D$ ) } \\
\hline Day 28 & $7.88 \pm 18.88$ & $-1.91 \pm 18.64$ & 0.0005 \\
\hline Day 42 & $1.32 \pm 18.31$ & $-5.09 \pm 17.02$ & 0.0154 \\
\hline \multicolumn{4}{|c|}{$\mathrm{FEV}_{1}$ (mean adjusted \% change from baseline) } \\
\hline Day 28 & 7.9 & -2.4 & $<0.0001$ \\
\hline Day 42 & 3.1 & -2.6 & 0.0024 \\
\hline \multicolumn{4}{|c|}{$\log _{10}$ PA cfu in sputum (mean adjusted change from baseline) } \\
\hline Day 28 & -1.384 & 0.069 & $<0.0001$ \\
\hline Day 42 & -0.078 & -0.010 & NS \\
\hline \multicolumn{4}{|c|}{ Required use of antipseudomonal antibiotics (\% of patients) } \\
\hline $\begin{array}{l}\text { IV or } \\
\text { additional inhaled } \\
\text { antibiotics }\end{array}$ & 15.0 & 22.6 & NS \\
\hline
\end{tabular}

Abbreviations: AZLI, aztreonam lysine for inhalation; cfu, colony-forming units; CFQ-R, CF Questionnaire-Revised; FEV , forced expiratory volume in one second; IV, intravenous; NS, nonsignificant; PA, Pseudomonas aeruginosa; tid, three times daily. predicted). Again, this population is older compared with studies reported with tobramycin inhalation solution. ${ }^{34,35}$ Additionally, this study only looked at efficacy following one treatment cycle. ${ }^{36}$

Data from an open-label follow-on study (AIR CF-3) have been published in abstract form. ${ }^{38-40}$ According to data released in the abstracts, 274 patients who originally took part in AIR CF-1 or AIR CF-2 were continued on up to nine 28-day on, 28-day off cycles of AZLI. AZLI was administered at a dose of $75 \mathrm{mg}$ twice daily or three times daily (as determined by the original study). Data suggest that improvements in disease-related endpoints $\left(\mathrm{FEV}_{1}\right.$, CFQ-R respiratory symptom score, and sputum $P$. aeruginosa density) generally persist over time during on-treatment cycles. Patients receiving AZLI three times daily had more favorable results as compared with those receiving AZLI twice daily ${ }^{38,39}$ However, owing to the lack of detail regarding study procedures, it is difficult to draw substantial conclusions at this time.

\section{Safety and tolerability}

The most commonly noted adverse events in Phase II and III clinical trials included cough, with a possible dose-related trend in incidence and severity, productive cough, wheezing, nasal congestion, headache, pharyngolaryngeal pain, pyrexia, and chest discomfort..$^{32,33,36}$ Airway reactivity after treatment, defined as an acute decrease in $\mathrm{FEV}_{1}$ by $15 \%$ or more within 30 minutes of nebulization, occurred in a similar number of patients in the treatment groups compared with placebo.

\section{Patient-focused perspectives}

While factors such as antimicrobial spectrum and pharmacokinetic parameters are important, patient-specific factors and perspectives, such as quality of life, must also be taken into consideration when determining the most appropriate therapy for treating pulmonary infections in patients with CF. Pulmonary infections are a significant source of morbidity and a reason for hospitalization among patients with CF. Use of antimicrobial strategies to minimize these complications can have a positive impact on the lives of these patients. However, multiple courses of systemic antibiotics have been associated with significant systemic side effects and appreciable collateral damage with respect to host flora. It has been these factors, as well as patient convenience, that have prompted clinicians to explore the use of inhaled antibiotics in this patient population. 
When compared specifically with intravenous antibiotics, inhaled antibiotics provide several advantages, including fewer invasive administration techniques, shorter administration times, fewer systemic adverse drug reactions, and reduced risk for administration-related infections. Furthermore, use of maintenance therapy with inhaled antibiotics may serve to help preserve pulmonary function among patients with CF more effectively than solely administering intravenous antibiotics in reaction to exacerbations of infections.

\section{Conclusion}

Use of inhaled antibiotics has long intrigued clinicians seeking a reliable means to deliver antimicrobials to the lungs. The premise has been that direct administration via nebulization would achieve high concentrations at the site of infection while minimizing systemic exposure. Although this approach is theoretically sound, its implementation has been fraught with problems, not the least of which has been the availability of drug products specifically formulated for aerosolized delivery. This barrier has been removed with the FDA approvals of tobramycin inhalation solution in 1997 and most recently aztreonam for inhalation in 2010, which in the US, is only available via a select network of specialty pharmacies. Each of these products was granted approval primarily as a result of data derived from research in patients with CF who were colonized/infected with $P$. aeruginosa. Each of these agents has demonstrated efficacy in reducing bacterial burdens, preserving lung function, and improving symptoms associated with disease..$^{33,34,36,41}$ Interestingly, a head-to-head comparative study funded by Gilead Sciences is currently underway examining the efficacies of tobramycin inhalation solution and AZLI. ${ }^{42}$

AZLI appears to be an active and safe therapeutic option. Studies have demonstrated a wide therapeutic window, with doses up to $225 \mathrm{mg}$ being well tolerated. ${ }^{31,32}$ Even at doses well below this threshold, aztreonam is effective in reducing bacterial loads in respiratory secretions. ${ }^{33,36}$ Additionally, there are data suggesting that aztreonam may maintain activity in the presence of sputum better than tobramycin. ${ }^{31}$ AZLI $75 \mathrm{mg}$ administered three times daily with the Altera ${ }^{\circledR}$ nebulizer system for 28 days on, 28 days off, is currently indicated to improve respiratory symptoms in CF patients seven years of age and older with $P$. aeruginosa and an FEV $25 \%-75 \%$ of predicted. ${ }^{24}$ Although approved for a wide patient subset, it is notable that the majority of clinical data reported to date have been generated in older patients with moderate lung disease.

Although AZLI and tobramycin inhalation solution are indicated for similar patient populations, it will be interesting to watch how clinicians utilize these agents. One important question that will need to be addressed is whether tobramycin inhalation solution and AZLI should be used as mono- or combination therapy. Additionally, if combination therapy is employed, should the agents be used as concurrent or cycled inhaled combinations? Another issue that will require further study is emergence of resistance. Given that tobramycin exhibits concentration-dependent killing, one might argue that some resistance may be overcome by the delivery of higher drug concentrations to the lungs. The value of higher concentrations of aztreonam in the lung in relation to long-term emergence of resistance remains to be determined. Also, it needs to be further elucidated which patient populations are most likely to benefit from AZLI administration. Data published to date have primarily examined patients with relatively stable and preserved lung function and have used exacerbation of pulmonary infection with $P$. aeruginosa as a clinical endpoint. Use in additional patient populations and for alternate indications needs to be established. It must also be remembered that no therapies have been shown to provide sustained eradication of $P$. aeruginosa.

One final consideration that may impact how readily AZLI is incorporated into clinical practice is the value added to patient care. The average wholesale price for a 28-day course of AZLI is approximately $\$ 5300$, and the average wholesale price for a 28-day course of tobramycin inhalation solution is approximately $\$ 4900$, both of which are significantly more expensive than aztreonam arginine. ${ }^{43,44} \mathrm{It}$ must then be asked whether this additional cost is justified in patient care. To this end, little data exist to support the notion that the specially formulated AZLI is significantly safer or more active than aztreonam arginine. However, the vast majority of clinical efficacy and safety data for nebulized administration of aztreonam to patients with $\mathrm{CF}$ have been generated using the aztreonam lysine formulation. Therefore, unless data prove otherwise, it would be prudent to let the evidence guide therapeutic decisions and utilize aztreonam lysine over aztreonam arginine when given via inhalation.

Clinical trials, experience, and cost may ultimately dictate how AZLI and other aerosolized antibiotics are used. However, for the time being, considerable interest exists regarding these agents. 


\section{Disclosure}

The authors report no conflicts of interest in this work.

\section{References}

1. Cystic Fibrosis Foundation. Cystic Fibrosis Foundation Patient Registry 2008 annual data report. Bethesda, MD: Cystic Fibrosis Foundation; 2009.

2. Flume PA, O'Sullivan BP, Robinson KA, et al. Cystic fibrosis pulmonary guidelines: Chronic medications for maintenance of lung health. Am J Respir Crit Care Med. 2007;176:957-969.

3. O'Sullivan BP, Freedman SD. Cystic fibrosis. Lancet. 2009;373: 1891-1904.

4. Montgomery GS, Howenstine M. Cystic fibrosis. Pediatr Rev. 2009;30: 302-309.

5. Gibson RL, Burns JL, Ramsey BW. Pathophysiology and management of pulmonary infections in cystic fibrosis. Am J Respir Crit Care Med. 2003;168:918-951.

6. Pamukcu A, Bush A, Buchdahl R. Effects of Pseudomonas aeruginosa colonization on lung function and anthropometric variables in children with cystic fibrosis. Pediatr Pulmonol. 1995;19:10-15.

7. Henry RL, Mellis CM, Petrovic L. Mucoid Pseudomonas aeruginosa is a marker of poor survival in cystic fibrosis. Pediatr Pulmonol. 1992;12: 158-161.

8. Emerson J, Rosenfeld M, McNamara S, Ramsey B, Gibson RL. Pseudomonas aeruginosa and other predictors of mortality and morbidity in young children with cystic fibrosis. Pediatr Pulmonol. 2002;34: 91-100.

9. Kerem E, Corey M, Gold R, Levison H. Pulmonary function and clinical course in patients with cystic fibrosis after pulmonary colonization with Pseudomonas aeruginosa. J Pediatr. 1990;116:714-719.

10. Flume PA, Robinson KA, O'Sullivan BP, et al. Cystic fibrosis pulmonary guidelines: Airway clearance therapies. Respir Care. 2009;54: 522-537.

11. Flume PA, Mogayzel PJ Jr, Robinson KA, et al. Cystic fibrosis pulmonary guidelines: Treatment of pulmonary exacerbations. Am J Respir Crit Care Med. 2009;180:802-808.

12. Emerson J, McNamara S, Buccat AM, Worrell K, Burns JL. Changes in cystic fibrosis sputum microbiology in the United States between 1995 and 2008. Pediatr Pulmonol. 2010;45:363-370.

13. Mendelman PM, Smith AL, Levy J, Weber A, Ramsey B, Davis RL. Aminoglycoside penetration, inactivation, and efficacy in cystic fibrosis sputum. Am Rev Respir Dis. 1985;132:761-765.

14. Levy J. Antibiotic activity in sputum. J Pediatr. 1986;108:841-846.

15. TOBI (Package Insert). East Hanover, NJ: Novartis Pharmaceuticals Corporation; 2010.

16. Ramphal R, Lhermitte M, Filliat M, Roussel P. The binding of antipseudomonal antibiotics to macromolecules from cystic fibrosis sputum. J Antimicrob Chemother. 1988;22:483-490.

17. Potter JL, Matthews LW, Spector S, Lemm J. Complex formation between basic antibiotics and deoxyribonucleic acid in human pulmonary secretions. Pediatrics. 1965;36:714-720.

18. Vaudaux P, Waldvogel FA. Gentamicin inactivation in purulent exudates: Role of cell lysis. J Infect Dis. 1980;142:586-593.

19. Geller DE, Konstan MW, Smith J, Noonberg SB, Conrad C. Novel tobramycin inhalation powder in cystic fibrosis subjects: Pharmacokinetics and safety. Pediatr Pulmonol. 2007;42:307-313.

20. Hagerman JK, Hancock KE, Klepser ME. Aerosolised antibiotics: A critical appraisal of their use. Expert Opin Drug Deliv. 2006;3: $71-86$.

21. Geller DE. Aerosol antibiotics in cystic fibrosis. Respir Care. 2009;54:658-670.

22. MacLeod DL, Barker LM, Sutherland JL, et al. Antibacterial activities of a fosfomycin/tobramycin combination: A novel inhaled antibiotic for bronchiectasis. J Antimicrob Chemother. 2009;64: $829-836$
23. Conrad D, Flume P, Sindel L, et al. Phase 2b study of inhaled MP-376 (Aeroquin, levofloxacin inhalation solution) in stable cystic fibrosis (CF) patients with chronic Pseudomonas aeruginosa (PA) lung infection. Am J Respir Crit Care Med. 2010;181:A2339.

24. Cayston (Package Insert). Foster City, CA: Gilead Sciences, Inc; 2010.

25. Azactam (Package Insert). Princeton, NJ: Bristol-Myers Squibb; 2010.

26. Dietzsch HJ, Gottschalk B, Heyne K, Leupoid W, Wunderlich P. Cystic fibrosis: Comparison of two mucolytic drugs for inhalation treatment (acetylcysteine and arginine hydrochloride). Pediatrics. 1975; 55:96-100.

27. Sapienza MA, Kharitonov SA, Horvath I, Chung KF, Barnes PJ. Effect of inhaled L-arginine on exhaled nitric oxide in normal and asthmatic subjects. Thorax. 1998;53:172-175.

28. Shawar RM, MacLeod DL, Garber RL, et al. Activities of tobramycin and six other antibiotics against Pseudomonas aeruginosa isolates from patients with cystic fibrosis. Antimicrob Agents Chemother. 1999;43: 2877-2880.

29. Bevivino A, Dalmastri C, Tabacchioni S, et al. Burkholderia cepacia complex bacteria from clinical and environmental sources in Italy: Genomovar status and distribution of traits related to virulence and transmissibility. J Clin Microbiol. 2002;40:846-851.

30. Berg G, Roskot N, Smalla K. Genotypic and phenotypic relationships between clinical and environmental isolates of Stenotrophomonas maltophilia. J Clin Microbiol. 1999;37:3594-3600.

31. Gibson RL, Retsch-Bogart GZ, Oermann C, et al. Microbiology, safety, and pharmacokinetics of aztreonam lysinate for inhalation in patients with cystic fibrosis. Pediatr Pulmonol. 2006;41:656-665.

32. Retsch-Bogart GZ, Burns JL, Otto KL, et al. A phase 2 study of aztreonam lysine for inhalation to treat patients with cystic fibrosis and Pseudomonas aeruginosa infection. Pediatr Pulmonol. 2008;43:47-58.

33. McCoy KS, Quittner AL, Oermann CM, Gibson RL, Retsch-Bogart GZ, Montgomery AB. Inhaled aztreonam lysine for chronic airway Pseudomonas aeruginosa in cystic fibrosis. Am J Respir Crit Care Med. 2008;178:921-928.

34. Ramsey BW, Pepe MS, Quan JM, et al. Intermittent administration of inhaled tobramycin in patients with cystic fibrosis. Cystic Fibrosis Inhaled Tobramycin Study Group. N Engl J Med. 1999;340:23-30.

35. Hagerman JK, Knechtel SA, Klepser ME. Tobramycin solution for inhalation in cystic fibrosis patients: A review of the literature. Expert Opin Pharmacother. 2007;8:467-475.

36. Retsch-Bogart GZ, Quittner AL, Gibson RL, et al. Efficacy and safety of inhaled aztreonam lysine for airway pseudomonas in cystic fibrosis. Chest. 2009;135:1223-1232.

37. Aztreonam for inhalation solution (NDA 50-814) for improvement of respiratory symptoms in cystic fibrosis patients. FDA briefing document for anti-infective drugs advisory committee meeting. December 10, 2009. Available at: http://www.fda.gov/ downloads/AdvisoryCommittees/CommitteesMeetingMaterials/ Drugs/Anti-InfectiveDrugsAdvisoryCommittee/UCM193023.pdf. Accessed May 24, 2010.

38. Oermann CM, Retsch-Bogart G, McCoy KS, Gibson R, Quittner AL, Montgomery B. Effect of multiple courses of aztreonam for inhalation solution (AZLI) on disease-related endpoints and safety in patients with CF: Final analysis of 18 month data [Abstr]. Pediatr Pulmonol. 2009; 44:296.

39. Oermann CM, McCoy KS, Retsch-Bogart GZ, Gibson R, McKevitt M, Montgomery B. Effect of repeated exposure to aztreonam for inhalation solution (AZLI) therapy on cystic fibrosis respiratory pathogens [Abstr]. Pediatr Pulmonol. 2009;44:335-336.

40. Oermann CM, McCoy KS, Retsch-Bogart GZ, Gibson R, McKevitt M, Montgomery B. Antibiotic susceptibility in Pseudomonas aeruginosa (PA) isolates following repeated exposures to aztreonam for inhalation solution (AZLI) in patients with cystic fibrosis [Abstr]. Pediatr Pulmonol. 2009;44:309.

41. Quittner AL, Buu A. Effects of tobramycin solution for inhalation on global ratings of quality of life in patients with cystic fibrosis and Pseudomonas aeruginosa infection. Pediatr Pulmonol. 2002;33: 269-276. 
42. Aztreonam for inhalation solution (AZLI) vs tobramycin inhalation solution $\left(\mathrm{TOBI}^{\circledR}\right)$ in patients with $\mathrm{CF}$ and P. aeruginosa. Available at: http:// clinicaltrials.gov/ct2/show/NCT00757237. Accessed May 25, 2010.

43. Specialty TrendsRx Alert. Cayston (aztreonam for inhalation solution). CVS Caremark, March 2010. Available at: https:/www.caremark.com/ portal/asset/SpecialtyTrendsRxAlert_Cayston.pdf. Accessed Jul 16, 2010 .
44. SRS Pharmacy Systems. Available at: www.srspharmacy.com. Accessed Jul 16, 2010.

\section{Publish your work in this journal}

Infection and Drug Resistance is an international, peer-reviewed openaccess journal that focuses on the optimal treatment of infection (bacterial, fungal and viral) and the development and institution of preventive strategies to minimize the development and spread of resistance. The journal is specifically concerned with the epidemiology of antibiotic resistance and the mechanisms of resistance development and diffusion in both hospitals and the community. The manuscript management system is completely online and includes a very quick and fair peerreview system, which is all easy to use. Visit http://www.dovepress.com/ testimonials.php to read real quotes from published authors.

Submit your manuscript here: http://www.dovepress.com/infection-and-drug-resistance-journal 